Case Report

\title{
Why It Is Not Always Anxiety: A Tough Diagnosis of Stiff Person Syndrome
}

\author{
Carmen Elena Cervantes, Hsien Lee Lau, Tina Ataian Binazir, \\ Keith O. O'Brien, and Jonathan S. Cross
}

Department of Medicine, Aventura Hospital and Medical Center, Aventura, FL, USA

Correspondence should be addressed to Carmen Elena Cervantes; carmen.cervantes@hcahealthcare.com

Received 20 April 2017; Revised 20 June 2017; Accepted 2 July 2017; Published 14 August 2017

Academic Editor: Isabella Laura Simone

Copyright (c) 2017 Carmen Elena Cervantes et al. This is an open access article distributed under the Creative Commons Attribution License, which permits unrestricted use, distribution, and reproduction in any medium, provided the original work is properly cited.

\begin{abstract}
Anxiety disorder is a commonly used diagnosis that may mask underlying conditions. Stiff person syndrome (SPS) is a rare neuroimmunological disorder characterized by progressive rigidity and painful muscle spasms affecting axial and lower extremity musculature. These episodes can be triggered by sudden movement, noise, or emotional stress, which may present as a psychiatric condition. We report the case of a 30-year-old female who presented with recurrent panic attacks with multiple prior hospital admissions for anxiety, rigidity, and difficulty in walking. Previous electroencephalogram (EEG) and brain and cervical spine magnetic resonance imaging (MRI) were unremarkable. She was empirically treated with diazepam and beta-blockers for SPS, which was confirmed by positive glutamic acid decarboxylase (GAD) antibodies. The patient's symptoms became refractory to benzodiazepines and required steroids with intravenous immunoglobulin (IVIG). Her rigidity subsequently responded to plasmapheresis. In SPS, antibodies in the cerebrospinal fluid (CSF) most commonly target the GAD antigen on gammaaminobutyric acid (GABA) neurons. The goal of treatment is to ameliorate symptoms and improve quality of life. Our case of SPS was masked as generalized anxiety disorder for at least six years since onset of symptoms. The criteria for both diagnoses may overlap as seen in this patient.
\end{abstract}

\section{Introduction}

Anxiety disorder is a commonly used diagnosis that may mask underlying conditions. Anxiety is mostly associated with rare diseases and is frequent among patients of some socioeconomic and cultural backgrounds. Stiff person syndrome (SPS) is a rare neuroimmunological disorder identified as an autoimmune or paraneoplastic syndrome [1]. It is characterized by progressive rigidity and painful muscle spasms affecting axial and lower extremity musculature. These episodes can be triggered by sudden movement, noise, or emotional stress, which may present as a psychiatric condition. Other cardinal symptoms include paroxysmal autonomic dysfunction with associated diaphoresis, tachycardia, pupillary dilatation, and hypertension [1-3]. Here, we report the case of a young patient previously diagnosed with anxiety disorder in whom years later SPS was identified.

A 30-year-old Haitian female with a past medical history of anxiety disorder and baseline tachycardia presented with recurrent panic attacks. The patient reported anxiety, palpitations, muscle stiffness, and painful spasms which started in the pelvis and radiated to bilateral lower extremities. Within a two-year period, she had multiple admissions for similar episodes of rigidity and difficulty in walking resulting in a diagnosis of anxiety. However, her first symptoms were reported six years previously. Prior to admission, she was empirically treated with diazepam and beta-blockers for SPS, while lab tests were pending.

On examination, vital signs were stable. Neurological exam revealed dystonia and tremors which were most apparent in bilateral lower extremities. Spasms were noted in all four limbs, as well as increased deep tendon reflexes: 3+ hyperreflexia in biceps, triceps, brachioradialis, and patellar and Achilles tendons. Cardiac and respiratory exams were unremarkable.

During previous hospital admissions, EEG did not detect ictal activity. Brain and cervical spine MRIs were unremarkable. Tests measuring creatinine kinase, aldolase, toxicology, 
TABLE 1: Diagnostic criteria for generalized anxiety disorder compared to Dalakas criteria for SPS.

\begin{tabular}{|c|c|}
\hline Generalized anxiety disorder criteria [1] & Dalakas criteria for SPS [4] \\
\hline \multicolumn{2}{|l|}{ (1) Excessive anxiety and worry, occurring more days than not for at least 6 months. } \\
\hline (2) The individual finds it difficult to control the worry. & (1) Stiffness in the axial muscles, particularly \\
\hline (3) The anxiety and worry are associated with three or more of the following: & abdominal and thoracolumbar paraspinal \\
\hline (a) Restlessness and feeling keyed up or on edge & muscles, leading to a fixed deformity: \\
\hline (b) Being easily fatigued & hyperlordosis. \\
\hline (c) Difficulty concentrating or mind ooino blank & (2) Superimposed painful spasms precipitated by \\
\hline (c) Difficulty concentrating or mind going blank & unexpected noises, emotional stress, or tactile \\
\hline (d) Irritability & stimuli. \\
\hline (e) Muscle tension & (3) Confirmation by electromyography (EMG). \\
\hline (f) Sleep disturbances & (4) Absence of neurological or cognitive \\
\hline $\begin{array}{l}\text { (4) Symptoms cause significant distress or impairment in social, occupational, or other } \\
\text { areas of functioning. }\end{array}$ & $\begin{array}{l}\text { (5) Positive serology: GAD or amphiphysin } \\
\text { autoantibodies. }\end{array}$ \\
\hline $\begin{array}{l}\text { (5) Symptoms are not attributable to the physiological effects of a substance or another } \\
\text { medical condition. }\end{array}$ & (6) Response to diazepam. \\
\hline
\end{tabular}

(1) Table adapted from DSM-V criteria for generalized anxiety disorder [5]. (2) Table adapted from Dalakas criteria for SPS [6]. Note. Text in italic represents symptoms/signs for the patient, meeting criteria for both GAD and SPS.

and thyroid function were within normal ranges. The patient's GAD antibodies were significantly elevated showing a level above 250 (normal: 0-5) units/mL.

The patient was initially treated with intravenous steroids and diazepam followed by IVIG for 3 days and finally improved with 5 cycles of plasmapheresis. The patient was discharged after 6 weeks with maintenance therapy of oral baclofen and diazepam.

\section{Discussion}

The distinguishing feature of our case is the presentation of SPS, concealed as an anxiety disorder. Stiff person syndrome is rare, with an estimated incidence of 1 per one million. It affects women 2-3 times more than men with an average age of presentation of 20-50 [1-3,6]. The duration from symptom onset to diagnosis ranges from 1 to 18 years, with a mean of 6.2 years [9].

The SPS diagnostic criteria are outlined in Table 1. Clinical manifestations have been categorized as classic SPS or variant SPS, such as stiff limb syndrome and progressive encephalomyelitis $[2,6,9]$. The stiffness and rigidity in classic SPS can be triggered by minimal physical or emotional stimuli, overtime causing increased anxiety and severe disability [6].

In SPS, antibodies in the CSF commonly target the GAD antigen on gamma-aminobutyric acid (GABA) neurons [1]. However, anti-GAD antibodies are present only in twothirds of patients $[6,9]$. GABA neurons inhibit spontaneous discharges from spinal motor neurons [1]. Therefore, their absence leads to a continuous activation of muscle motor units. It is unclear whether the disease process underlying SPS is directly responsible for anxiety disorders or whether anxiety disorders develop as a reaction to disability associated with SPS. A recent neuropsychological cohort study of 10 patients with SPS and symptoms of anxiety suggested that anxiety was a reaction to disability. Low levels of GABA lead to an overall higher firing frequency of nerve cells, which can manifest as either anxiety or even seizure disorders. This may lower the threshold for overactivity of the amygdala, the fear and anxiety center of the brain [10].

When analyzed, criteria for both disorders may overlap as represented in Table 1 . The text in italics specifically shows which conditions our patient met from both diseases. She met all criteria for anxiety disorder, except for the fifth component: the underlying medical condition which was not initially identified. For SPS, she met all the conditions, except for EMG which was not performed. With regard to demographics, she fits two features of both conditions: age group of 20-50 years and female gender.

The goal of treatment is to improve quality of life by providing symptom relief. Benzodiazepines are considered first-line treatment in SPS $[8,10]$. As a $\mathrm{GABA}_{\mathrm{A}}$ agonist, diazepam is used for its muscle relaxant and anxiolytic properties. Baclofen is used orally with diazepam for its $\mathrm{GABA}_{B}$ agonist activity to manage spasticity $[1,6,8]$. Table 2 outlines more treatment options.

This case yields insight into the similarities between SPS and anxiety which may delay diagnosis and management for an internist or general neurologist. Anxiety and functional disorders presenting with recurrent muscular rigidity and/or episodic autonomic symptoms resembling panic attacks should raise alarm of an alternative neurologic disease. Eating disorders, hysterical paralysis, phobias, posttraumatic stress disorder, and agoraphobia have been misdiagnosed when SPS was the underlying condition [11]. Psychogenic movement disorder is the most important differential diagnosis of SPS. It is characterized by a normal neurological examination (including a normal blink reflex test), distractibility, and symptom improvement without significant interventions [12]. Hysterical paralysis and other conversion disorders rarely persist for more than 1 year, while SPS will have a more progressive course $[13,14]$. By continuing to broaden our understanding and knowledge of the diverse presentations 
TABLE 2: Treatment options for stiff person syndrome.

\begin{tabular}{ll}
\hline Type of treatment & Treatment description \\
First-line treatment & (i) Benzodiazepines \\
Other GABAergic drugs & (ii) Diazepam is used both as an anticonvulsant and as a muscle relaxant, with oral baclofen \\
Oral versus intrathecal & (i) Gabapentin, tiagabine, and levetiracetam have been used for reduction in symptoms \\
baclofen & (i) Oral baclofen with diazepam is part of the first-line treatment for GABA agonist activity \\
Treatment with & (ii) Intrathecal baclofen has been used for severe spasticity and has shown significant improvement \\
intravenous & (i) IVIG (2 g/kg over two to five days) is used for symptomatic relief when first-line treatment fails \\
immunoglobulin versus & and patients have severe disability in carrying out daily activities \\
plasmapheresis & (ii) Plasmapheresis showed promising results in $56 \%$ of patients when first-line therapy failed [7] \\
Rituximab & (i) Monoclonal antibody that binds to the B-lymphocyte cluster of differentiation surface antigen \\
& (ii) Administration: 2 doses are infused with spacing of 7-14 days or as four weekly infusions \\
& (iii) Improves gait when benzodiazepines and monthly IVIG treatment have failed
\end{tabular}

Modified from Ariño et al. [8].

of SPS, we can decrease the morbidity associated with longstanding misdiagnosis.

\section{Conflicts of Interest}

The authors declare that there are no financial conflicts of interest.

\section{References}

[1] T. Chang, H. Alexopoulos, M. McMenamin et al., "Neuronal surface and glutamic acid decarboxylase autoantibodies in nonparaneoplastic stiff person syndrome," JAMA Neurology, vol. 70, no. 9, pp. 1140-1149, 2013.

[2] S. Hadavi, A. J. Noyce, R. D. Leslie, and G. Giovannoni, "Stiff person syndrome," Practical Neurology, vol. 11, no. 5, pp. 272282, 2011.

[3] M. B. Pagano, B. B. Murinson, A. A. R. Tobian, and K. E. King, "Efficacy of therapeutic plasma exchange for treatment of stiffperson syndrome," Transfusion, vol. 54, no. 7, pp. 1851-1856, 2014.

[4] A. B. Bhatti and Z. A. Gazali, "Recent advances and review on treatment of stiff person syndrome in adults and pediatric patients," Cureus, vol. 7, no. 12, p. e427, 2015.

[5] J. F. Baizabal-Carvallo and J. Jankovic, "Stiff-person syndrome: insights into a complex autoimmune disorder," Journal of Neurology, Neurosurgery \& Psychiatry, vol. 86, no. 8, pp. 840848, 2015.

[6] M. C. Dalakas, "Stiff person syndrome: advances in pathogenesis and therapeutic interventions," Current Treatment Options in Neurology, vol. 11, no. 2, pp. 102-110, 2009.

[7] American Psychiatric Association, Diagnostic and Statistical Manual of Mental Disorders, American Psychiatric Association, Washington, DC, USA, 2013.

[8] H. Ariño, R. Höftberger, N. Gresa-Arribas et al., "Paraneoplastic neurological syndromes and glutamic acid decarboxylase antibodies," JAMA Neurology, vol. 72, no. 8, pp. 874-881, 2015.

[9] M. C. Dalakas, M. Fujii, M. Li, and B. McElroy, "The clinical spectrum of anti-GAD antibody-positive patients with stiffperson syndrome," Neurology, vol. 55, no. 10, pp. 1531-1535, 2000 .
[10] H. Tohid, "Anti-glutamic acid decarboxylase antibody positive neurological syndromes," Neurosciences, vol. 21, no. 3, pp. 215222, 2016.

[11] B. C. McLeod, I. Sniecinski, D. Ciavarella et al., "Frequency of immediate adverse effects associated with therapeutic apheresis," Transfusion, vol. 39, no. 3, pp. 282-288, 1999.

[12] M. Cuturic, L. M. Harden, M. H. Kannaday, N. N. Campbell, and R. K. Harding, "Stiff-person syndrome presenting as eating disorder: A case report," International Journal of Eating Disorders, vol. 44, no. 3, pp. 284-286, 2011.

[13] C. S. H. Ho, R. C. M. Ho, and E. P. Wilder-Smith, "Stiff person syndrome masquerading as panic attacks," The Lancet, vol. 383, no. 9917, article 668, 2014.

[14] D. Fleischman, G. Madan, T. A. Zesiewicz, and M. Fleischman, "Stiff-person syndrome: Commonly mistaken for hysterical paralysis," Clinical Neurology and Neurosurgery, vol. 111, no. 7, p. 644, 2009. 


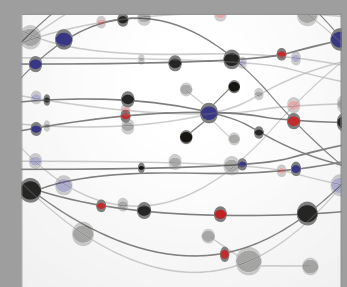

The Scientific World Journal
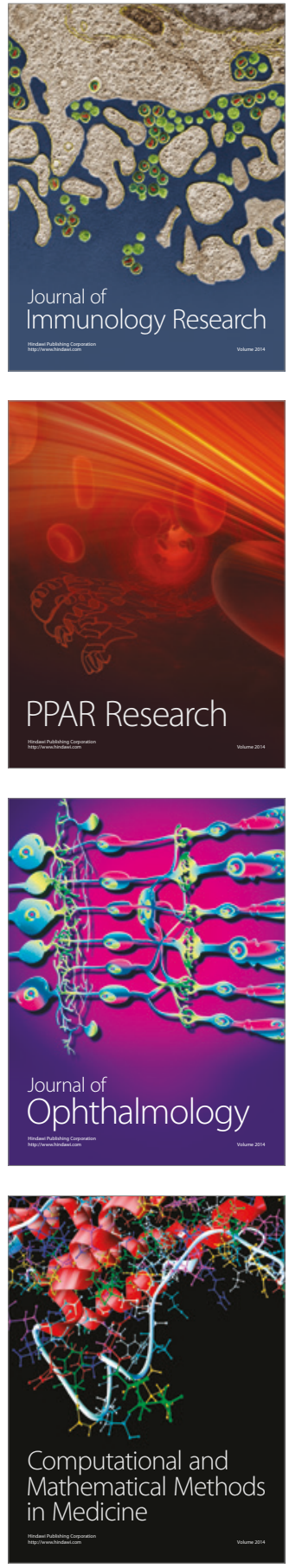

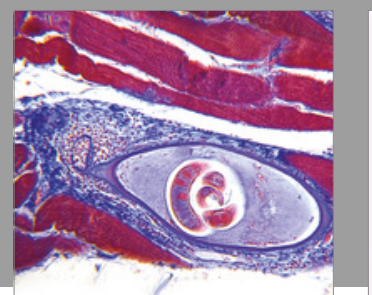

Gastroenterology Research and Practice
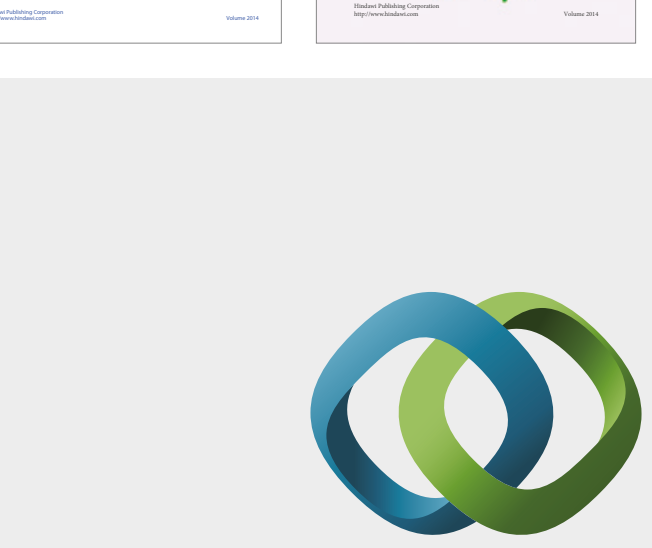

\section{Hindawi}

Submit your manuscripts at

https://www.hindawi.com
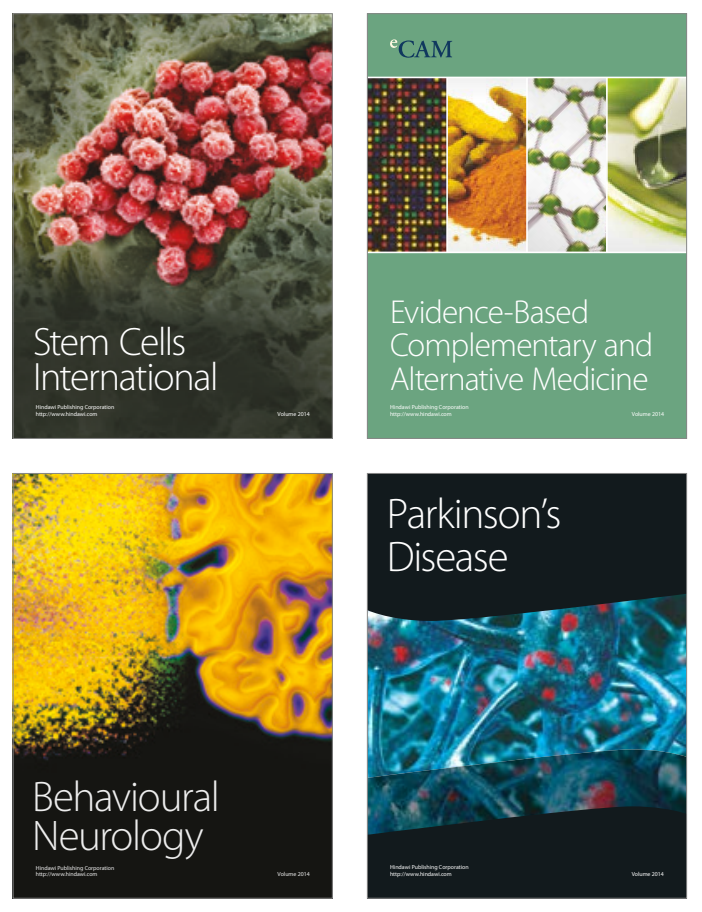
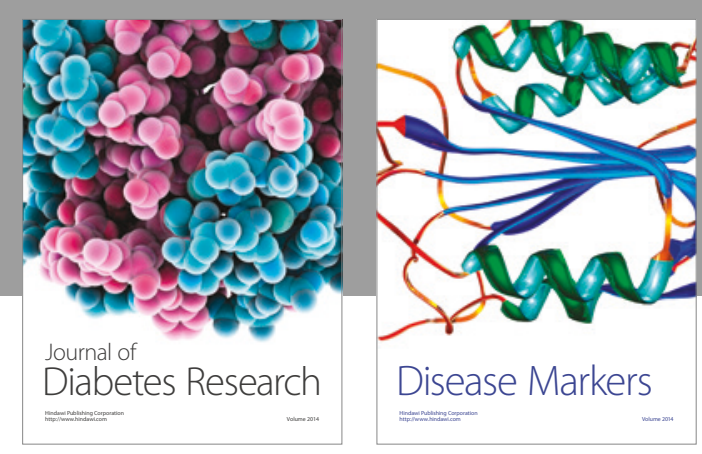

Disease Markers
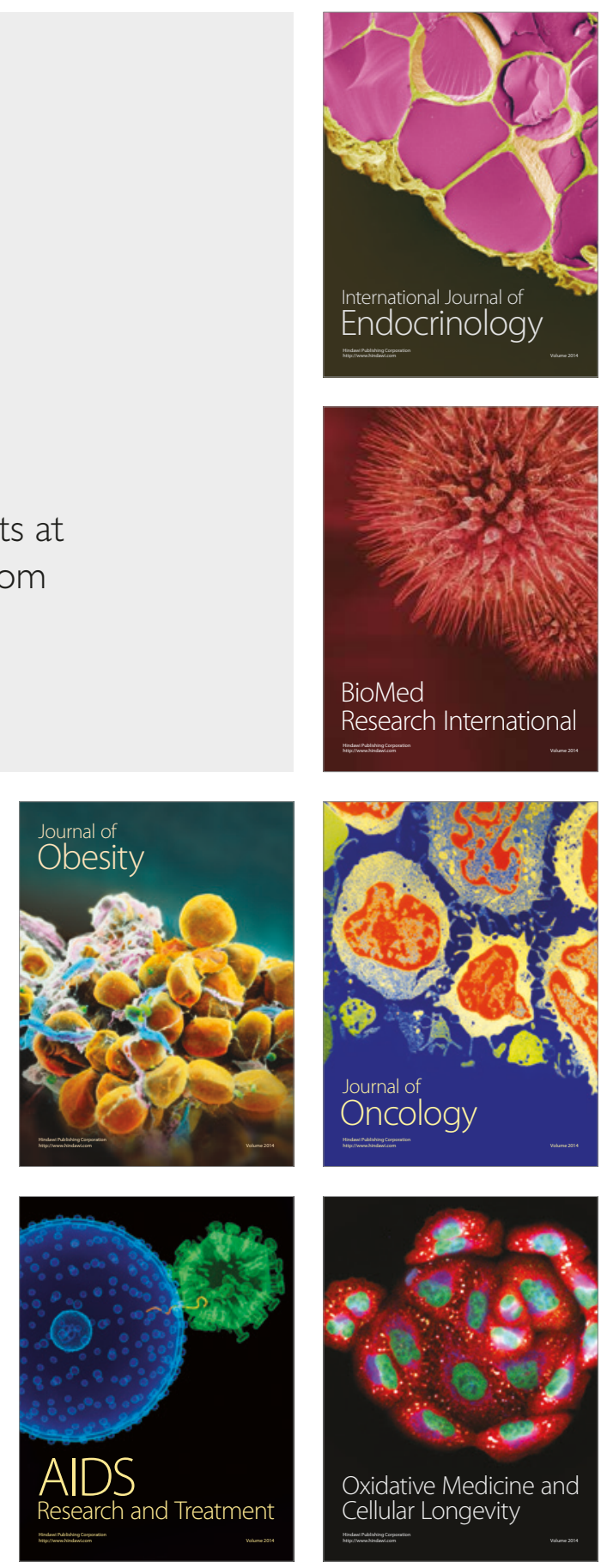